\title{
T2 Relaxometry MRI Predicts Cerebral Palsy in Preterm Infants
}

\author{
(1DL.-W. Chen, DS.-T. Wang, (1DC.-C. Huang, (1) Y.-F. Tu, and (1) Y.-S. Tsai
}

\begin{abstract}
BACKGROUND AND PURPOSE: T2-relaxometry brain MR imaging enables objective measurement of brain maturation based on the water-macromolecule ratio in white matter, but the outcome correlation is not established in preterm infants. Our study aimed to predict neurodevelopment with T2-relaxation values of brain MR imaging among preterm infants.
\end{abstract}

MATERIALS AND METHODS: From January 1, 2012, to May 31, 2015, preterm infants who underwent both T2-relaxometry brain MR imaging and neurodevelopmental follow-up were retrospectively reviewed. T2-relaxation values were measured over the periventricular white matter, including sections through the frontal horns, midbody of the lateral ventricles, and centrum semiovale. Periventricular $\mathrm{T} 2$ relaxometry in relation to corrected age was analyzed with restricted cubic spline regression. Prediction of cerebral palsy was examined with the receiver operating characteristic curve.

RESULTS: Thirty-eight preterm infants were enrolled for analysis. Twenty patients (52.6\%) had neurodevelopmental abnormalities, including 8 (21\%) with developmental delay without cerebral palsy and $12(31.6 \%)$ with cerebral palsy. The periventricular T2-relaxation values in relation to age were curvilinear in preterm infants with normal development, linear in those with developmental delay without cerebral palsy, and flat in those with cerebral palsy. When MR imaging was performed at $>1$ month corrected age, cerebral palsy could be predicted with $\mathrm{T} 2$ relaxometry of the periventricular white matter on sections through the midbody of the lateral ventricles (area under the receiver operating characteristic curve $=0.738$; cutoff value of $>217.4$ with $63.6 \%$ sensitivity and $100.0 \%$ specificity).

CONCLUSIONS: T2-relaxometry brain MR imaging could provide prognostic prediction of neurodevelopmental outcomes in premature infants. Age-dependent and area-selective interpretation in preterm brains should be emphasized.

ABBREVIATIONS: BSID-II = Bayley Scales of Infant and Toddler Development, 2nd ed; BSID-III = Bayley Scales of Infant and Toddler Development, 3rd ed; CP = cerebral palsy; $\mathrm{DD}=$ delayed development; $\mathrm{ROC}=$ receiver operating characteristic curve

$\mathbf{P}$ remature infants are at high risk of cognitive and behavior problems. It remains a challenging issue in $50 \%-60 \%$ of extremely preterm infants despite the advances in neonatal critical care. ${ }^{1}$ Although late preterm infants born between 33 and 37 weeks of gestational age are considered low-risk, the

Received July 12, 2017; accepted after revision October 30

From the Departments of Pediatrics (L.-W.C., C.-C.H., Y.-F.T.) and Diagnostic Radiology (Y.-S.T.), National Cheng Kung University Hospital, College of Medicine, National Cheng Kung University, Tainan, Taiwan; Institutes of Clinical Medicine (L.-W.C.) and Gerontology (S.-T.W.), College of Medicine, National Cheng Kung University, Tainan, Taiwan; and Department of Pediatrics (C.-C.H.), Taipei Medical University, College of Medicine, Taipei, Taiwan.

This work was supported by National Cheng Kung University Hospital-10403006.

Please address correspondence to Yi-Shan Tsai, MD, Department of Diagnostic Radiology, National Cheng Kung University Hospital, College of Medicine, National Cheng Kung University, 138 Sheng-Li Rd, Tainan City 70403, Taiwan; e-mail: crevice123@hotmail.com

-- Indicates open access to non-subscribers at www.ajnr.org

http://dx.doi.org/10.3174/ajnr.A5501 neurodevelopmental scales still fall behind compared with term controls. ${ }^{2}$

MR imaging is considered a good tool for early diagnosis of brain injury in prematurity, but the conventional qualitative approach remains subjective. ${ }^{3,4}$ The incidence of macroscopic cystic-necrotic periventricular leukomalacia has markedly declined, and new forms of microscopic focal or diffuse nondestructive white matter injury are emerging. ${ }^{5}$ However, this microscopic necrosis and gliosis is not readily detected by conventional MR imaging. ${ }^{6}$

T2 relaxometry, also called T2 mapping, is a quantitative T2-weighted MR imaging that demonstrates the physical properties of brain-water, enabling objective measurement of tissue characteristics based on the water-macromolecule ratio in white matter. In very preterm infants, $\mathrm{T} 2$ relaxometry has been applied to discriminate diffuse excessive high signal intensity in white matter at term-equivalent age. ${ }^{7}$ However, the changes of T2 relaxometry in preterm periventricular WM injury and 


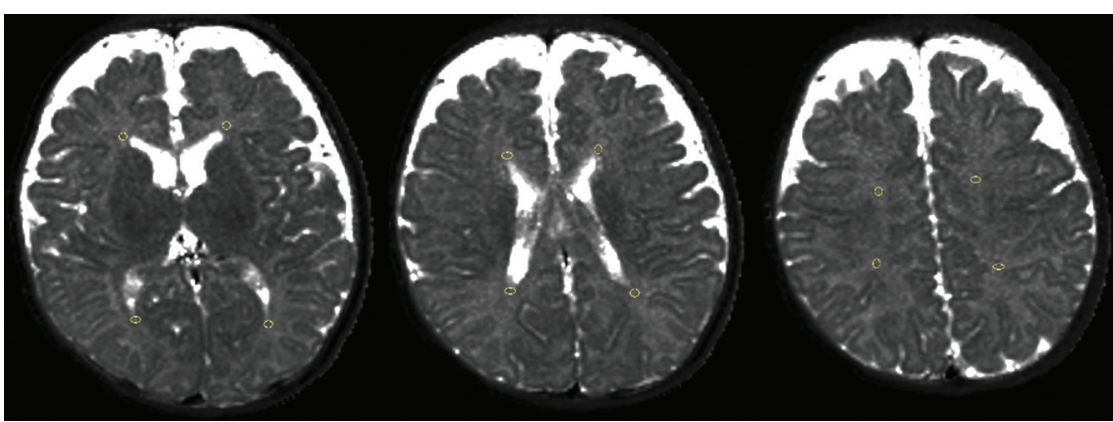

FIG 1. Manually drawn periventricular white matter on 3 axial sections through the frontal horns, midbody of the lateral ventricles, and centrum semiovale. The circular ROI area is $5-8 \mathrm{~mm}^{2}$. The 4 T2-mapping values of each section are averaged for calculation. centrum semiovale, ROIs were placed over 2 sites in each hemisphere. The 4 T2-mapping values of each section were averaged. When there were cystic lesions in the periventricular white matter, the ROIs were placed over the nearby parenchymal tissue rather than the fluid component within the cysts. The measurements were performed twice by 1 pediatric neurologist and once by 1 radiologist, generating intrarater and interrater reliabilities. Data of the 3 measurements were averaged for final analyses. further correlation to neurodevelopmental outcomes have not been established. ${ }^{4,7}$

In contrast to the physiologic decrease of cerebral water in healthy infants, WM injury leading to delayed myelination and tissue destruction may present with free water retention and prolong the T2 value on brain MR imaging. ${ }^{8}$ Our study hypothesizes that there are distinct patterns of $\mathrm{T} 2$ relaxometry according to the neurodevelopmental status. Furthermore, this study provides a model of outcome prediction with T2-relaxometry brain MR imaging in premature infants.

\section{MATERIALS AND METHODS \\ Participants}

From January 1, 2012, to May 31, 2015, preterm infants (born at $<37$-week gestational age) who underwent T2-relaxometry brain MR imaging at younger than 1 year of age were included. The demographic data and clinical indications of brain MR imaging were retrospectively reviewed. We excluded patients with major neurologic underlying diseases, such as congenital cerebral malformation, hypoxic-ischemic encephalopathy, shaken baby syndrome, and neonatal/infantile epilepsy. Patients who had neurodevelopmental follow-up for $>12$ months in the hospital were enrolled for analysis. The institutional review board of National Cheng Kung University Hospital approved the study.

\section{Neuroimaging Studies}

The patients were scanned on an Achieva 1.5T MR imaging scanner (Philips Healthcare, Best, the Netherlands). The infants were swaddled and administered oral chloral hydrate (50 mg per kilogram) before MR imaging. The pulse sequences included axial T1WI, axial T2WI, FLAIR, DWI, and T2 relaxometry. T2 relaxometry was performed using an ultrafast turbo gradient spinecho sequence (TSE factor, 5; TR/TE, 3500/20, 40, 60, 80, 100 ms; twenty-eight 4-mm-thick axial sections without a slice gap; FOV, $200 \times 200 \mathrm{~mm}^{2}$; matrix, $224 \times 200$; voxel size, $0.9 \times 1 \mathrm{~mm}$; bandwidth, $73.7 \mathrm{~Hz}$ /pixel; acquisition time 4 minutes 50 seconds).

Three sections of periventricular white matter, including axial sections through the frontal horns, midbody of the lateral ventricles, and centrum semiovale, were selected for analyses (Fig 1).9,10 A circular ROI with 5- to 8- $\mathrm{mm}^{2}$ area was positioned for measurement. For sections through the frontal horns and midbody of the lateral ventricles, ROIs were placed over the periventricular white matter of the anterior and posterior ventricular poles. For the

\section{Neurodevelopmental Outcomes}

Neurodevelopmental evaluations were performed at the outpatient clinics by pediatric neurologists, who decided the eligibility for tests with the Bayley Scales of Infant and Toddler Development, 2nd ed (BSID-II) or 3rd ed (BSID-III). Delayed development (DD) was defined with a motor or language scale of $<70$ with the BSID-II or a cognitive, motor, or language scale of $<85$ with the BSID-III. ${ }^{11}$ Cerebral palsy (CP), apart from the pure DD, was defined as severe impairment of motor function with spasticity due to nonprogressive disturbances in the brain. ${ }^{12}$

\section{Statistical Analysis}

The T2-quantification values of periventricular white matter in relation to age were fitted with semi-log plots and further analyzed with restricted cubic spline regression in preterm infants with normal development, DD without $\mathrm{CP}$, and CP. The comparison between the $\mathrm{CP}$ and non-CP groups was performed with the $\chi^{2}$ test for categoric variables and the Mann-Whitney $U$ test for numeric variables, evaluating the differences in average attenuation values and the adjusted attenuation values. To predict $\mathrm{CP}$ with $\mathrm{T} 2$ relaxometry, we assessed the logistic regression with the backward selection method and the receiver operating characteristic (ROC) curve. ${ }^{13}$ Cut-point estimation, 95\% confidence interval, and areas under the ROC curve were calculated. Sensitivities and specificities at cutoff points were determined with the Youden index. The level of significance was set at $P<.05$. The intrarater reliability was represented with the intraclass correlation, which was rated as fair at $0.40-0.59$, good at $0.60-0.74$, and excellent when $>0.74 .{ }^{14}$ The interrater reliability was represented with the interrater agreement $\kappa$ coefficient and was defined as moderate at $0.41-0.60$, good at $0.61-0.80$, and very good at $0.81-1.00 .^{15}$

Statistical analysis was performed using commercially available software (MedCalc for Windows, Version 14.8.1; MedCalc Software, Mariakerke, Belgium.) The age-versus-T2quantification graphs and semi-log lines were plotted with GraphPad Prism 5 software (GraphPad Software, San Diego, California). Restricted cubic spline regression and prediction models of CP were performed with SAS statistical software (SAS Institute, Cary, North Carolina). 
PV Frontal

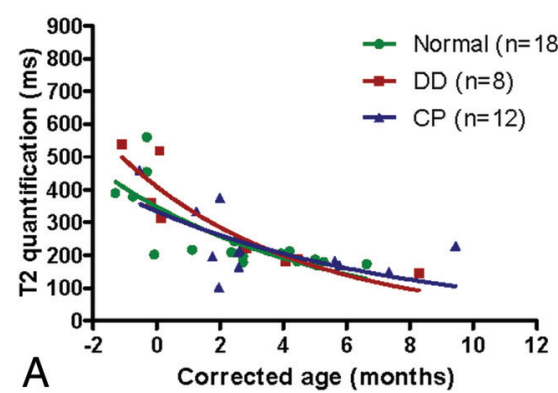

PV Midbody

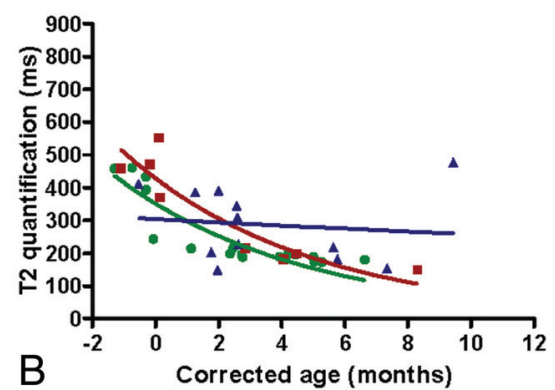

Centrum Semiovale

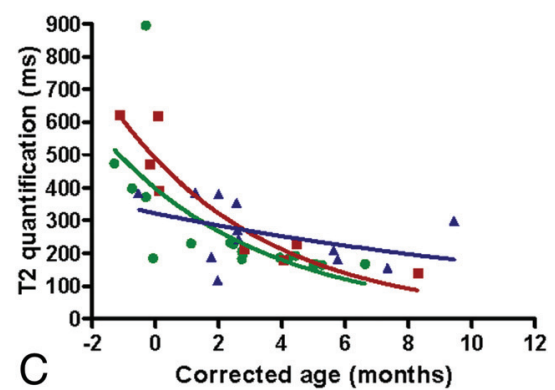

FIG 2. T2-relaxation values versus age among patients with normal development, delayed development without cerebral palsy, and cerebral palsy. T2 quantification in periventricular white matter declines with age. Periventricular white matter through sections of the frontal horns of the lateral ventricles $(A)$, midbody of the lateral ventricles $(B)$, and centrum semiovale $(C)$. DD indicates delayed development (without cerebral palsy); PV, periventricular.

\section{RESULTS}

During the study period, 57 preterm infants underwent 60 brain MR imaging examinations with T2 relaxometry before 1 year of age. For patients who had $>1$ brain MR imaging, only the first study was analyzed. Nineteen patients were excluded because of congenital cerebral malformation $(n=6)$, neonatal/infantile epilepsy $(n=5)$, loss to follow-up $(n=4)$, hypoxic-ischemic encephalopathy $(n=2)$, shaken baby syndrome $(n=1)$, and poor imaging quality $(n=1)$. Thus, 38 patients were enrolled for analysis.

The average gestational age was $31.8 \pm 3.4$ weeks (range, $24.0-$ 37.0 weeks), and the mean birth weight was $1675.7 \pm 771.4 \mathrm{~g}$ (range, 505-3554 g). Among the 38 preterm infants, 7 were girls and 31 were boys. Five patients had brain MR imaging due to CNS symptoms, and the other 33 patients without clinical symptoms had brain MR imaging for abnormalities on screening brain sonography. CNS symptoms included isolated seizure $(n=2)$, CNS infection $(n=1)$, facial palsy $(n=1)$, and precocious puberty $(n=1)$. Abnormalities on screening brain sonography included persistent periventricular hyperechogenicity $(n=18)$, periventricular leukomalacia $(n=7)$, ventriculomegaly $(n=6)$, porencephalic cyst $(n=1)$, and absence of the septum pellucidum $(n=1)$.

Neurodevelopment was followed for a mean of $31.7 \pm 9.9$ months (range, 12.0-50.2 months). Among the 38 patients, 29 were evaluated with the BSID-III, and 3, with the BSID-II. The BSID examinations were performed at a mean age of $21.1 \pm 7.1$ months; (range, 7.0-34.0 months). The remaining 6 patients, for whom BSID tests were not arranged, were confirmed as having normal neurodevelopmental statuses by pediatric neurologists during scheduled visits. Among the 38 patients, 20 (52.6\%) had developmental disorders, including 8 (21\%) with pure DD without CP and 12 (31.6\%) with CP.

Brain MR imaging was performed at a mean of 2.8-month corrected age, ranging from 1.3 months before term to 9.4 months of age. The intrarater correlation coefficients corresponding to periventricular white matter ranged from 0.73 to 0.78 , indicating good-toexcellent intraobserver reliability. The interrater agreement coefficients among regions of white matter ranged from 0.57 to 0.73 , suggesting moderate-to-good reliability between the 2 observers.

The age-dependent curve of T2-relaxometry values versus corrected age is shown in Fig 2. In infants with normal neurode-

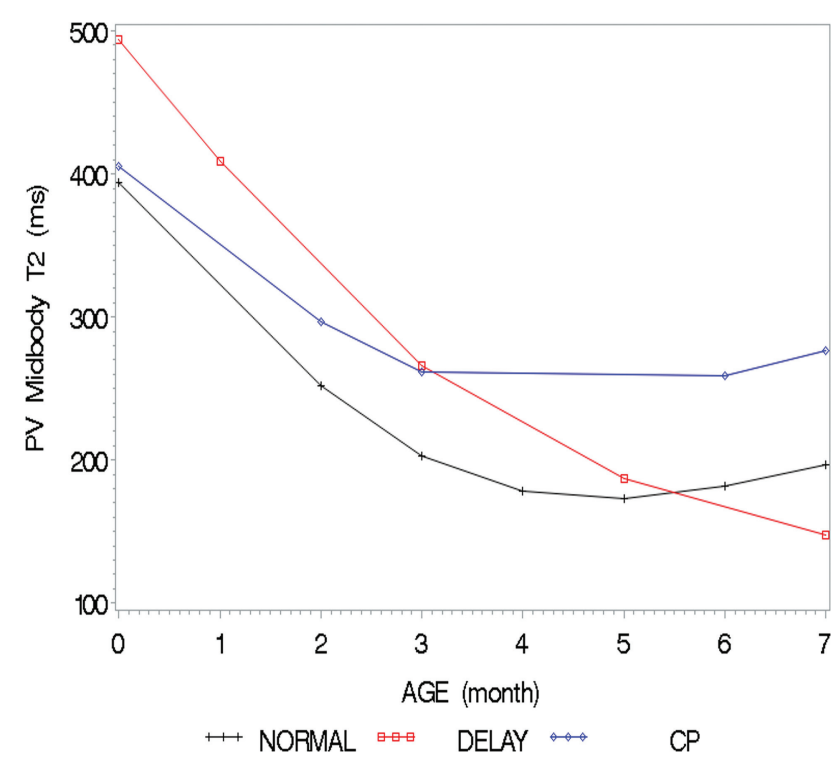

FIG 3. Restricted cubic spline regression analysis of $T 2$ relaxometry versus age in the midbody periventricular white matter. T2-quantification values in relation to age are curvilinear, linear, and flat among normal development (black line), delayed development (red line), and cerebral palsy (blue line) in preterm infants, respectively. PV indicates periventricular.

velopment and patients with $\mathrm{DD}$, T2-relaxometry values decreased with age. However, the T2-quantification values in the $\mathrm{CP}$ group did not show a rapid decline, especially in the periventricular white matter around the midbody of the lateral ventricles and centrum semiovale. Further analysis with restricted cubic spline regression showed that $\mathrm{T} 2$ relaxation values at the periventricular white matter in relation to age were curvilinear in normal development, linear in DD, and flat in CP (Fig 3).

For age-specific analysis of CP prediction, patients were reclassified into 2 groups according to MR imaging before or after 1 -month corrected age (Table). ${ }^{16,17}$ There was only 1 patient who developed CP among the 10 neonates who underwent MR imaging examinations before 1 month of age. T2 mapping had no statistically significant impact on prediction of CP in this group. Twenty-eight patients had MR imaging performed after 1-month corrected age (mean corrected age of MR imaging, $4.2 \pm 2.1$ months), and there were 11 patients diagnosed with $\mathrm{CP}$. There was no difference in sex, gestational age, birth body weight, and 
Demographic data of birth history, birth condition, and $\mathrm{T} 2$ relaxometry values at 3 different sections of periventricular white matter ${ }^{\mathrm{a}}$

\begin{tabular}{|c|c|c|c|c|c|}
\hline & \multicolumn{2}{|c|}{$\leq 1 \mathrm{~m} / \circ$ CGA at MRI $(n=10)$} & \multicolumn{3}{|c|}{$>1 \mathrm{~m} / \circ$ CGA at MRI $(n=28)$} \\
\hline & Non-CP & $\mathrm{CP}$ & Non-CP & $\mathrm{CP}$ & $P$ Value \\
\hline No. & 9 & 1 & 17 & 11 & \\
\hline Sex, M/F & 9:0 & 1:0 & $12: 5$ & $9: 2$ & .823 \\
\hline GA (wk) & $32.3 \pm 3.1$ & 37.0 & $31.9 \pm 3.4$ & $30.9 \pm 3.6$ & .397 \\
\hline BBW (gm) & $1586.6 \pm 746.4$ & 2160 & $1863.3 \pm 862.8$ & $1411.1 \pm 675.0$ & .158 \\
\hline DOIC (+) (No.) & $4 / 9$ & 0 & $9 / 17$ & $1 / 8$ & .137 \\
\hline $\operatorname{PROM}(+)($ No. $)$ & $2 / 9$ & 0 & $5 / 17$ & $1 / 8$ & .673 \\
\hline 1st Apgar' score & $4.6 \pm 2.5$ & 9 & $4.9 \pm 2.4$ & $6.3 \pm 1.5$ & .217 \\
\hline 2nd Apgar ${ }^{b}$ score & $6.7 \pm 2.2$ & 10 & $7.7 \pm 2.1$ & $8.2 \pm 0.8$ & .800 \\
\hline CGA at MRI (mo) & $-0.4 \pm 0.5$ & -0.5 & $4.0 \pm 1.7$ & $3.9 \pm 2.7$ & .410 \\
\hline PV frontal & $412.5 \pm 116.5$ & 458.8 & $194.8 \pm 24.1$ & $211.1 \pm 79.7$ & .814 \\
\hline PV midbody & $426.4 \pm 85.2$ & 410.8 & $191.3 \pm 16.9$ & $226.8 \pm 110.6$ & $.036^{c}$ \\
\hline Centrum semiovale & $491.3 \pm 201.0$ & 382.6 & $186.9 \pm 28.0$ & $252.8 \pm 92.5$ & .086 \\
\hline
\end{tabular}

Note:- $\mathrm{m}$ /o indicates month old; CGA, corrected gestational age; GA, gestational age; BBW, birth body weight; DOIC, delay of initial crying; PROM, premature rupture of membrane; PV, periventricular.

${ }^{\text {a }}$ Data are mean \pm SD unless otherwise indicated.

${ }^{b}$ The Apgar score is an assessment of overall newborn well-being.

${ }^{c} P$ value $<.05$.

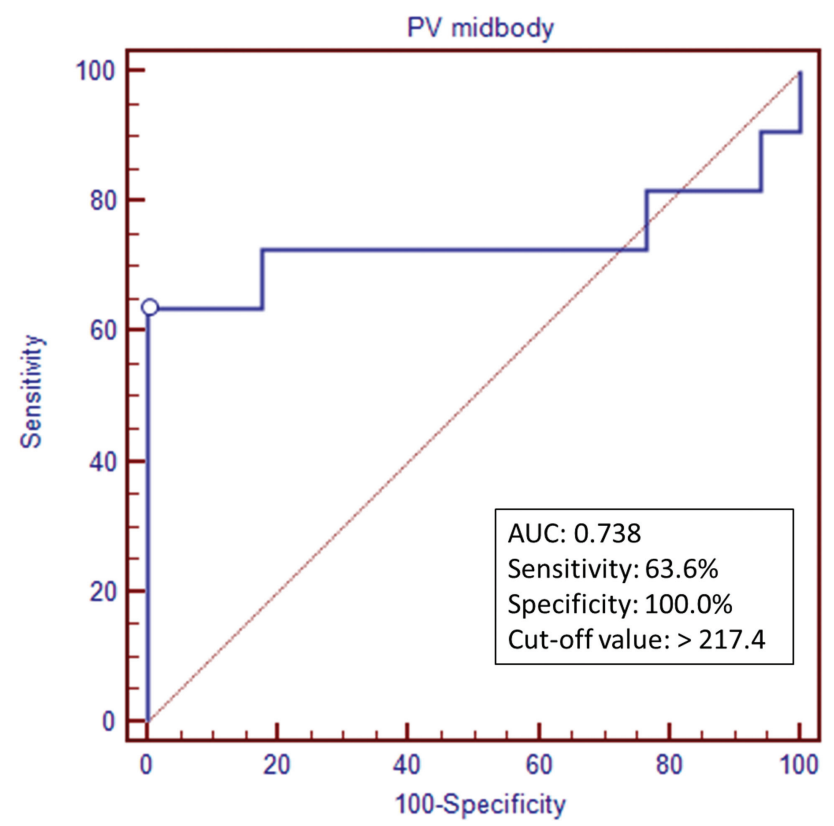

FIG 4. Receiver operating characteristic curves of $T 2$ relaxometry on the sections through the midbody of the lateral ventricles.

birth condition between the patients with and without $\mathrm{CP}$ in this group. The periventricular T2-relaxometry value around the midbody of the lateral ventricles was significantly higher in patients with $\mathrm{CP}(P=.036)$. The area under the ROC curve for predicting $\mathrm{CP}$ with T2-relaxation values in the midbody periventricular white matter was 0.738 (95\% CI, 0.538-0.885) with ROC analysis. The sensitivity and specificity for diagnosing CP using the optimized cutoff point of the T2 relaxometry value $(>217.4)$ were $63.6 \%$ and $100 \%$, respectively (Fig 4).

\section{DISCUSSION}

With the advances of neonatal intensive care, macroscopic cystic periventricular leukomalacia has been replaced by other forms of white matter lesions, including microscopic necrosis and diffuse white matter injury, both of which are prone to be underestimated in conventional qualitative MR imaging. ${ }^{18} \mathrm{~T} 2$ relaxometry is a fast scan to quantify tissue characteristics, providing an objec- tive measurement of the watery contents of the brain. ${ }^{4}$ Our study found that white matter maturation patterns were different depending on the neurologic outcomes because T2 relaxometry showed curvilinear decline in preterm infants with normal development, linear decline in those with DD, and was flat in those with CP.

T2-relaxation values descended rapidly in the first few months due to the fast myelination process during the first year of life. ${ }^{19,20}$ This rapid decline of $\mathrm{T} 2$ relaxometry observed in normal brain maturation corresponded to the curvilinear decline in our preterm infants with normal development. In this study, the altered $\mathrm{T} 2$ relaxometry patterns in the CP group illustrated slower decline of T2 quantification, with relatively lower $\mathrm{T} 2$ values in the early stage of WM injury and higher T2 values in the later stage.

The T2-relaxation values in periventricular WM in 2.4T MR imaging ranged from 214 to $247 \mathrm{~ms}$ when healthy premature infants were scanned at 37 weeks of postconceptional age, suggesting the watery characteristics of brain tissue in premature infants at the perinatal stage. ${ }^{21}$ In our study of infants with CP, the damaged WM revealed lower $\mathrm{T} 2$ relaxation than the normal watery WM in the neonatal period and higher T2 relaxation values than the normal myelinated WM in later infancy. Previous animal studies found that T2WI hypointensity in the early stage of cerebral WM injury was related to pathologic microcysts and diffuse noncystic gliosis, and our observation of lower $\mathrm{T} 2$ values at the early stage of WM injury in infants with CP may reflect the pathologic changes. ${ }^{22,23}$

During a normal developmental process, the anterior caps and posterior periventricular bands in the deep white matter around the lateral ventricles are associated with the denseness of migrating neurons and white matter fibers converging from different regions of the hemisphere. ${ }^{24}$ The T2WI signals could vary according to the neuronal cells and fiber composition; however, all these caps and periventricular bands should not be obvious after 40 weeks of gestational age. ${ }^{10,25}$ Furthermore, the brain free water in the deep cerebral white matter starts to decrease physiologically after 1 month of life. ${ }^{17}$ As a result, to diminish the effects of caps/ periventricular bands and water decline interferences, we regrouped the patients according to MR imaging before or after 1 -month corrected age for analysis of CP prediction. 
In our observation, T2-relaxation values of the midbody periventricular white matter could predict $\mathrm{CP}$ when MR imaging was performed after 1-month corrected age. In preterm infants, $\mathrm{T} 2$ relaxometry of $>217.4$ predicted $\mathrm{CP}$ with $63.6 \%$ sensitivity and $100.0 \%$ specificity. When predicting outcomes with the signals on T2-quantification MR imaging, infants' ages should be considered cautiously. Although not a very early neuroimaging indicator compared with other studies using term-equivalent age MR imaging, ${ }^{3}$ our T2-quantification model could still enhance the clinical judgment in high-risk preterm infants because the diagnosis of cerebral palsy might sometimes be equivocal before 18 months of age. ${ }^{12}$

The study was limited by the retrospective design and small sample size. Prediction of outcomes with MR imaging before 1-month corrected age could not be achieved because of the small study population. In the study, only preterm infants who had clinical indications for brain MR imaging were included; thus, there was selection bias resulting in the higher rates of $\mathrm{CP}$ and DD in our series. ${ }^{12}$ The significance of T2 quantification in preterm cerebral development should be further verified through universal studies in both high- and low-risk preterm infants. The tools of developmental evaluation in the study included BSID-II and BSID-III. Although there was discrepancy between the 2 editions, the correlation of BSID-II and BSID-III in preterm infants has been addressed in other investigations. ${ }^{11}$ The analyses of developmental performance with categoric outcomes further excluded the direct comparison between BSID-II and BSID-III scores.

In our work, T2 relaxometry was estimated with 5 TEs (20, 40, $60,80,100 \mathrm{~ms}$ ); however, the maximum TE of $100 \mathrm{~ms}$ may not be long enough for accurate measurements in premature infants' brains. The value for the T2 relaxometry would be underestimated when using few echoes and an insufficient TE. For better estimation, previous studies suggested that 32 echoes with multiexponential fits were optimal to define the T2-decay curve; however, the long scanning time may be a restriction for young infants. ${ }^{26,27}$ The precision of T2-relaxometry measurements could be diminished due to partial volume effects because the spatial resolution was limited in the study. The T2-quantification values might be expanded because of mixed periventricular white matter and adjacent CSF within the ROIs. Early quantitative MR imaging in thin slices with autocalculation computer programs may provide more precise prediction than the results in our study. ${ }^{3,28}$ However, the simple manual measurement of T2 relaxometry in specific white matter areas could still be useful clinically.

Our study focused on the neuroimaging-neurodevelopmental correlations, and the clinical events regarding the perinatal complications were not analyzed. Further comprehensive studies of T2-quantification MR imaging in a large population of preterm infants could provide more illustrative information on the interaction of perinatal clinical events, neuroimaging variations, and long-term neurobehavioral outcomes.

\section{CONCLUSIONS}

T2-relaxometry brain MR imaging could be of prognostic value in preterm infants. The maturation patterns of periventricular white matter differed according to neurodevelopmental outcomes. T2 relaxation values over the midbody periventricular white matter at $>1$-month corrected age could predict CP. T2-relaxometry brain MR imaging provides neuroimaging-outcome correlation among preterm infants, especially when interpreted with age-specific and area-selective considerations.

\section{ACKNOWLEDGMENTS}

We thank Mr Feng-Mao Chiu, the clinical MR imaging application specialist of Philips Healthcare, for the support in the simulation model in T2-estimation accuracy.

\section{REFERENCES}

1. Kidokoro H, Anderson PJ, Doyle LW, et al. Brain injury and altered brain growth in preterm infants: predictors and prognosis. Pediatrics 2014;134:e444-53 CrossRef Medline

2. Romeo DM, Brogna C, Sini F, et al. Early psychomotor development of low-risk preterm infants: influence of gestational age and gender. Eur J Paediatr Neurol 2016;20:518-23 CrossRef Medline

3. Plaisier A, Raets MM, Ecury-Goossen GM, et al. Serial cranial ultrasonography or early MRI for detecting preterm brain injury? Arch Dis Child Fetal Neonatal Ed 2015;100:F293-300 CrossRef Medline

4. He L, Parikh NA. Atlas-guided quantification of white matter signal abnormalities on term-equivalent age MRI in very preterm infants: findings predict language and cognitive development at two years of age. PLoS One 2013;8:e85475 CrossRef Medline

5. Ment LR, Hirtz D, Hüppi PS. Imaging biomarkers of outcome in the developing preterm brain. Lancet Neurol 2009;8:1042-55 CrossRef Medline

6. Pierson CR, Folkerth RD, Billiards SS, et al. Gray matter injury associated with periventricular leukomalacia in the premature infant. Acta Neuropathol 2007;114:619-31 CrossRef Medline

7. Hagmann CF, De Vita E, Bainbridge A, et al. T2 at MR imaging is an objective quantitative measure of cerebral white matter signal intensity abnormality in preterm infants at term-equivalent age. $R a$ diology 2009;252:209-17 CrossRef Medline

8. Ashikaga R, Araki Y, Ono Y, et al. Appearance of normal brain maturation on fluid-attenuated inversion-recovery (FLAIR) MR images. AJNR Am J Neuroradiol 1999;20:427-31 Medline

9. He L, Parikh NA. Automated detection of white matter signal abnormality using T2 relaxometry: application to brain segmentation on term MRI in very preterm infants. Neuroimage 2013;64:328-40 CrossRef Medline

10. Maalouf EF, Duggan PJ, Rutherford MA, et al. Magnetic resonance imaging of the brain in a cohort of extremely preterm infants. $J \mathrm{Pe}$ diatr 1999;135:351-57 CrossRef Medline

11. Moore T, Johnson S, Haider S, et al. Relationship between test scores using the second and third editions of the Bayley Scales in extremely preterm children. J Pediatr 2012;160:553-58 CrossRef Medline

12. Spittle AJ, Orton J. Cerebral palsy and developmental coordination disorder in children born preterm. Semin Fetal Neonatal Med 2014; 19:84-89 CrossRef Medline

13. Steyerberg EW, Vickers AJ, Cook NR, et al. Assessing the performance of prediction models: a framework for traditional and novel measures. Epidemiology 2010;21:128-38 CrossRef Medline

14. Fleiss, JL. Statistical Methods for Rates and Proportions. 2nd ed. New York: Wiley; 1981

15. Altman DG. Practical Statistics for Medical Research. London: Chapman and Hall/CRC; 1990

16. Welker KM, Patton A. Assessment of normal myelination with magnetic resonance imaging. Semin Neurol 2012;32:15-28 CrossRef Medline

17. Murakami JW, Weinberger E, Shaw DW. Normal myelination of the pediatric brain imaged with fluid-attenuated inversion-recovery (FLAIR) MR imaging. AJNR Am J Neuroradiol 1999;20:1406-11 Medline

18. Back SA. Brain injury in the preterm infant: new horizons for 
pathogenesis and prevention. Pediatr Neurol 2015;53:185-92 CrossRef Medline

19. Ding XQ, Kucinski T, Wittkugel O, et al. Normal brain maturation characterized with age-related $\mathrm{T} 2$ relaxation times: an attempt to develop a quantitative imaging measure for clinical use. Invest $R a-$ diol 2004;39:740 - 46 CrossRef Medline

20. Leppert IR, Almli CR, McKinstry RC, et al; Brain Development Cooperative Group. T(2) relaxometry of normal pediatric brain development. J Magn Reson Imaging 2009;29:258 - 67 CrossRef Medline

21. Ferrie JC, Barantin L, Saliba E, et al. MR assessment of the brain maturation during the perinatal period: quantitative T2 MR study in premature newborns. Magn Reson Imaging 1999;17: 1275-88 CrossRef Medline

22. Riddle A, Dean J, Buser JR, et al. Histopathological correlates of magnetic resonance imaging-defined chronic perinatal white matter injury. Ann Neurol 2011;70:493-507 CrossRef Medline

23. Jeon TY, Kim JH, Yoo SY, et al. Neurodevelopmental outcomes in preterm infants: comparison of infants with and without diffuse excessive high signal intensity on MR images at near-term-equivalent age. Radiology 2012;263:518-26 CrossRef Medline

24. Childs AM, Ramenghi LA, Evans DJ, et al. MR features of developing periventricular white matter in preterm infants: evidence of glial cell migration. AJNR Am J Neuroradiol 1998;19:971-76 Medline

25. Girard N, Raybaud C, Poncet M. In vivo MR study of brain maturation in normal fetuses. AJNR Am J Neuroradiol 1995;16: 407-13 Medline

26. Whittall KP, MacKay AL, Graeb DA, et al. In vivo measurement of T2 distributions and water contents in normal human brain. Magn Reson Med 1997;37:34-43 CrossRef Medline

27. Whittall KP, MacKay AL, Li DK. Are mono-exponential fits to a few echoes sufficient to determine $\mathrm{T} 2$ relaxation for in vivo human brain? Magn Reson Med 1999;41:1255-57 Medline

28. Sled JG, Nossin-Manor R. Quantitative MRI for studying neonatal brain development. Neuroradiology 2013;55(suppl 2):97-104 CrossRef Medline 